\title{
Stellar Yields of Rotating First Stars
}

\author{
Koh Takahashi* \\ University of Tokyo \\ E-mail: ktakahashieastron.s.u-tokyo.ac.jp
}

\section{Hideyuki Umeda}

University of Tokyo

E-mail: umedadastron.s.u-tokyo.ac.ip

\section{Takashi Yoshida}

Kyoto University

E-mail: voshidaeyukawa.kyoto-u.ac.jp

\begin{abstract}
Metal poor stars provide valuable information about nucleosynthesis of the early universe. Interestingly, three known hyper metal-poor stars showing extremely low iron abundance of $[\mathrm{Fe} / \mathrm{H}]$ $<-5$ similarly have some peculiarity in their abundance patterns, i.e., they show enhancement of intermediate mass elements such as $\mathrm{C}, \mathrm{N}, \mathrm{O}, \mathrm{Na}$, and $\mathrm{Mg}$. We calculate stellar evolution of massive Population III stars, and compare the stellar yields with the observed abundance patterns of hyper metal-poor stars. We show that the stellar yields depend on the initial parameters such as initial mass and initial rotation, and show that the resulted yields can explain the observations. We conclude that the peculiar abundance patterns of intermediate mass elements observed in hyper metal-poor stars will provide possible constraints on distributions of the initial mass and rotation of the Population III stars.
\end{abstract}

XIII Nuclei in the Cosmos

7-11 July, 2014

Debrecen, Hungary

\footnotetext{
* Speaker.
} 


\section{Introduction}

Stars that firstly formed in the universe are called first stars or Population III (Pop III) stars. They initiated the cosmic reionization emitting high energy photons, and the elements synthesized by this Pop III stars were ejected and firstly contributed to the chemical evolution of the universe.

How can we infer the characteristics of the first objects which existed in the early universe long time ago? A good candidate for the investigation is to use abundance patterns preserved in metal-poor stars. Metal-poor stars should be born in a metal-poor gas cloud. Since a metal pollution history of such a gas is simple, the metal abundance is expected to show nucleosynthesis features of the metal supplying mother star(s). Therefore, the abundance patterns have been used to constrain theoretical models for nucleosynthesis in the mother progenitor stars, and the method is called abundance profiling.

Until now, several works investigated yields from the Pop III stars and the first supernovae. Explosive yields of heavy isotopes were the main focus in these works, and some important features of the first supernovae, such as the energetics and the asphericity, has been constrained. On the other hand, no abundance features that can be used to constrain the initial parameters of Pop III progenitors are known until now. Recently, thanks to the development of star formation theory, some interesting information on initial stellar state has been known. For example, Hirano et al. [四] showed that the initial mass of Pop III stars can be in the wide range from $\sim 10 \mathrm{M}_{\odot}$ to $\sim 1000 \mathrm{M}_{\odot}$. Also, Stacy et al. [] ] found that a Pop III star can have a fast rotation velocity at its birth. Thus, the aim of this work is to find useful features of stellar yields by doing abundance profiling, from which the initial mass and the rotation of the first stars can be constrained.

\section{Method}

\subsection{Stellar Evolution Calculation}

We calculate the evolution of massive zero metallicity stars using the latest version of our stellar evolution code [目]. A wide mass range from $12 \mathrm{M}_{\odot}$ to $140 \mathrm{M}_{\odot}$ is taken as the initial masses in order to cover a likely mass range for all core collapse supernovae. Also, stellar rotation is included in the calculation, which affects the yields by effective internal mixing due to several rotational instabilities. Two cases of models, with and without rotation, are calculated for each initial mass.

\subsection{Assumptions on Supernova Explosion}

Stellar matter is assumed to be ejected by supernova explosion at the end of the stellar life. Since the explosion and ejection mechanisms are complicated to be consistently taken into account, some assumptions and approximations are applied. We consider the case of energetically weak explosions, and call the explosion as a weak supernova. The first assumption is that only weakly-bound outer part of the star is ejected by the explosion. And another assumption is that the explosion is too weak to modify the composition of outer distribution of matter by shock heating. With these assumptions, stellar yields $M_{i}$ can be calculated by a simple integration with the 
parameter for the inner boundary $M_{i n}$,

$$
M_{i}\left(M_{i n}\right)=\int_{M_{\text {in }}}^{\mathrm{M}_{\text {surface }}} X_{i}(M) d M
$$

where $X_{i}$ is a mass fraction distribution of $i$ element. A parameter $f_{i n}=M_{i n} / M_{\mathrm{CO}}$ is also used in the analysis, here $M_{\mathrm{CO}}$ is a mass of the $\mathrm{CO}$ core.

\subsection{Abundance Calculation}

The chemical composition of a second generation star can be calculated as

$$
X_{i, 2 \mathrm{nd}}=\frac{M_{i} / M_{\mathrm{SN}}+X_{i, \mathrm{ISM}} M_{\mathrm{ISM}} / M_{\mathrm{SN}}}{1+M_{\mathrm{ISM}} / M_{\mathrm{SN}}}
$$

where $M_{\mathrm{SN}}=\sum_{i} M_{i}$ and $M_{\mathrm{ISM}}$ are total masses of the ejecta and the inter stellar matter (ISM), and $D=M_{\mathrm{ISM}} / M_{\mathrm{SN}}$ is a dilution factor. Dilution factors vary with models, and typically become several hundreds for best fitted models for hyper metal-poor stars. In the calculation, we assume that the ejected material is fully mixed with the ISM, and the second generation star is formed in the inter stellar gas cloud. Comparison between calculated abundances and observation is done by using $[\mathrm{i} / \mathrm{j}]$ as the indicator, calculated as

$$
[i / j]=\log \left(\frac{X_{i, 2 \mathrm{nd}}}{X_{j, 2 \mathrm{nd}}}\right)-\log \left(\frac{X_{i, \odot}}{X_{j, \odot}}\right) .
$$

\section{Stellar Yields}

Figure $\mathbb{W}$ shows calculated abundance distribution in models of rotating and non-rotating, 30 and $80 \mathrm{M}_{\odot}$ stars. Important features of abundance distribution, which are appeared in outer regions of stars are summarized below.

Carbon and Oxygen All stellar models yield carbon and oxygen in their helium layers. These elements are formed by triple alpha reaction and ${ }^{12} \mathrm{C}(\alpha, \gamma){ }^{16} \mathrm{O}$ reaction. Since abundant helium remains during the evolution in this region, resulting $\mathrm{C} / \mathrm{O}$ ratio becomes large and always exceeds unity. Carbon and oxygen production with a large $[\mathrm{C} / \mathrm{O}]$ can be regarded as a general signature of nucleosynthesis in the outer region of a star.

Magnesium and Silicon In the figure, magnesium and silicon are produced in $80 \mathrm{M}_{\odot}$ models, and generally, these alpha elements are produced only in massive models. This is because the production sequence of alpha capture reactions requires high temperature at the base of the helium layer, which is favored by more massive models. Production and non-production of these alpha elements are, thus, useful to constrain the initial mass of the progenitor.

Sodium and Aluminum Both rotating 30 and $80 \mathrm{M}_{\odot}$ models yield sodium and aluminum in their helium layers, while non-rotators do not. This is due to the rotationally induced mixing in the rotating models. First, carbon and oxygen synthesized by core helium burning are transported into hydrogen burning shell by the rotationally induced mixing. This results in nitrogen production by $\mathrm{CNO}$ cycle, and nitrogen distributes in the helium layer. Then alpha capture reactions on nitrogen 
take place, producing ${ }^{22} \mathrm{Ne}$, a famous neutron source for massive star evolution. Finally, neutron capture reactions on ${ }^{22} \mathrm{Ne}$ and ${ }^{26} \mathrm{Mg}$ take place, producing ${ }^{23} \mathrm{Na}$ and ${ }^{27} \mathrm{Al}$. Because of this, sodium and aluminum enhancement can be used to constrain the stellar rotation of the progenitor.

Calcium Only very massive models yield calcium, and the mechanisms are different between rotating and non-rotating progenitors. For rotating models, efficient alpha capture reactions are responsible for the production, and the process is similar to the production of magnesium and silicon. For non-rotating models, efficient proton capture reactions take place at the base of the hydrogen burning shell and account for the calcium production.

\section{Abundance Profiling}

In this section, we describe how best fit models can be determined in the abundance profiling of three hyper metal-poor stars. Comparison between observed abundances and theoretical models are shown in Fig. $\square$, and results of abundance profiling are summarized in Table $\square$.

SMSS 0313-6708 SMSS 0313-6708, recently found by [B]], is the most iron-deficient star known until now. Iron has not been detected in the star, and very small upper limit of $[\mathrm{Fe} / \mathrm{H}]<-7$ has been reported. Interesting feature of the composition of the star is the enhancement of carbon $([\mathrm{C} / \mathrm{H}] \sim$ $-2.6)$ and magnesium $([\mathrm{Mg} / \mathrm{H}] \sim-3.9)$. In addition, upper limits of $[\mathrm{Na} / \mathrm{Mg}]<-1.2$ and $[\mathrm{Al} / \mathrm{Mg}]<$ -1.9 are valuable for the abundance profiling.

First, massive stars of $>100 \mathrm{M}_{\odot}$ stars overproduce magnesium at helium layers. These stars fail to explain the small $[\mathrm{Mg} / \mathrm{C}]$ and thus rejected as the progenitor star. On the other hand, less
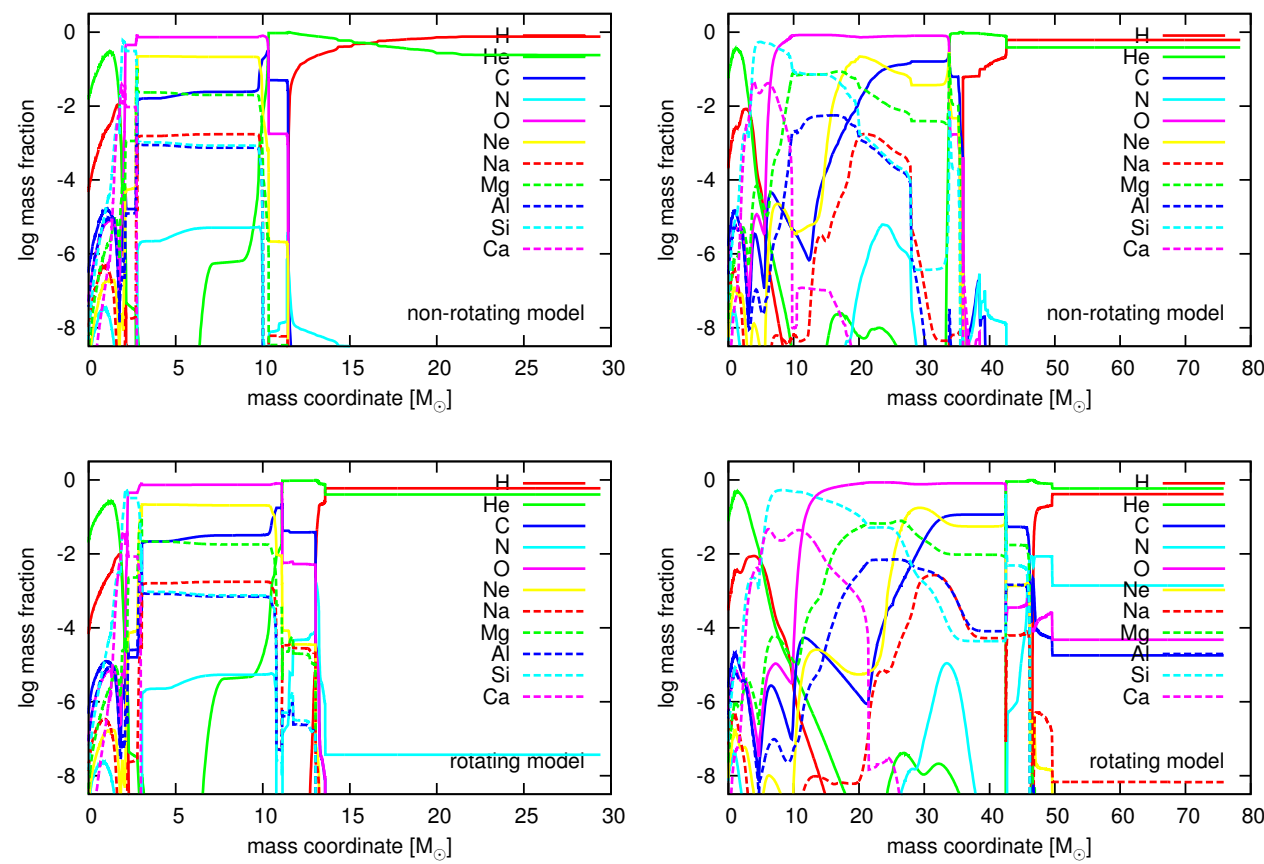

Figure 1: Abundance distributions of rotating and non-rotating, 30 and $80 \mathrm{M}_{\odot}$ models. 
massive models of $<40 \mathrm{M}_{\odot}$ produce sodium and aluminum besides magnesium, and do not fit with the observation. Similarly, the upper limits of sodium and aluminum are used to constrain rotating models, since all rotating progenitors produce sodium and aluminum in helium layers due to the rotationally induced mixing. Therefore, a massive, but not too massive, non-rotating star can be a progenitor of SMSS 0313-6708. The non-rotating $60 \mathrm{M}_{\odot}$ gives the best fitting to the abundance pattern from carbon to silicon. Calcium has been detected in the star with a small value of $[\mathrm{Ca} / \mathrm{H}]=-$ 7.0. If future observations reveal the enhancement of calcium as well as other intermediate mass elements, then the non-rotating $80 \mathrm{M}_{\odot}$ will be a good candidate for the progenitor, since the model produces calcium by fast proton capture reactions.

HE 1327-2326 and HE 0107-5240 The other two iron-deficient stars, HE 1327-2326 ([Fe/H]=5.7, reported by [] $]$ ) and HE 0107-5240 ([Fe/H]=-5.3, reported by [四]), are also compared with

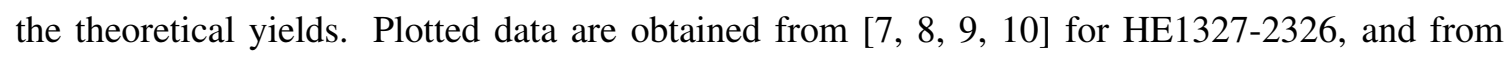
[11, [2], [3]], and for 3D collection from [14]].

Abundance pattern from sodium to aluminum is useful to constraint the progenitor of $\mathrm{HE}$ 1327-2326. The pattern, and the relative abundance with carbon (e.g., $[\mathrm{Mg} / \mathrm{C}]=-2.7$ ) can be well represented by ejecting tiny fraction of material in the carbon burning region of $\sim 15-40 \mathrm{M}_{\odot}$ models. Thus, rotating $15-30 \mathrm{M}_{\odot}$ and non-rotating $20-40 \mathrm{M}_{\odot}$ models give good fits for HE 13272326. HE 0107-5240 has a highly enhanced carbon abundance relative to oxygen, $[\mathrm{O} / \mathrm{C}]=-1.4$. Such a pattern can only be explained by a mass ejection from a very outer region of the progenitor star. In addition, sodium abundance is much larger than magnesium abundance in the star, $[\mathrm{Na} / \mathrm{Mg}]$ $=0.8$, and stellar rotation can be responsible for the enhancement. In our models, the rotating 30
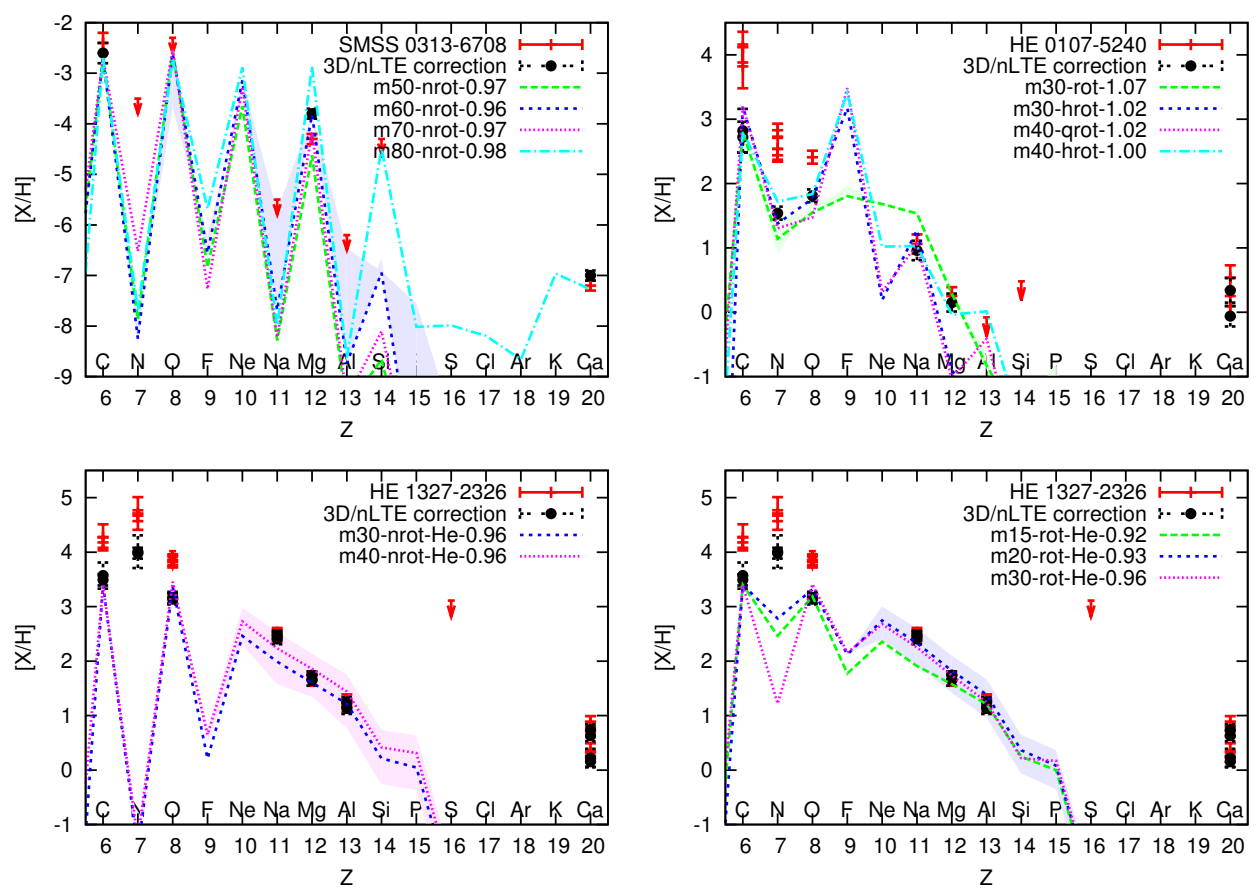

Figure 2: Results of abundance profiling. 
Table 1: Summary of the abundance profiling.

\begin{tabular}{cccccc}
\hline Object & {$[\mathrm{Fe} / \mathrm{H}]$} & $M_{\text {ini }}$ & $f_{\text {in }}$ & Rotation & Dilution Factor \\
\hline SMSS 0313-6708 & $<-7.1$ & $50-80$ & $0.96 \pm 0.04\left(60 \mathrm{M}_{\odot}\right)$ & non-rotating & $1.78 \times 10^{3}-6.09 \times 10^{2}$ \\
& & & $0.98 \pm 0.04\left(80 \mathrm{M}_{\odot}\right)$ & non-rotating & $1.62 \times 10^{3}-1.91 \times 10^{2}$ \\
HE 0107-5240 & -5.3 & $30-40$ & $1.07 \pm 0.06\left(30 \mathrm{M}_{\odot}\right)$ & rotating & $7.84 \times 10^{2}-2.23 \times 10^{2}$ \\
HE 1327-2326 & -5.7 & $20-40$ & $0.96 \pm 0.01\left(40 \mathrm{M}_{\odot}\right)$ & non-rotating & $5.00 \times 10^{2}-4.32 \times 10^{2}$ \\
& & $15-30$ & $0.93 \pm 0.01\left(20 \mathrm{M}_{\odot}\right)$ & rotating & $7.92 \times 10^{2}-7.35 \times 10^{2}$ \\
\hline
\end{tabular}

$\mathrm{M}_{\odot}$ star with $f_{\text {in }}=1.01-1.13$ gives the best fit to the star.

\section{Conclusion}

We find that intermediate mass elements such as carbon, nitrogen, sodium, and magnesium, differently distribute in an outer region of massive Pop III stars, depending on the initial mass and initial rotational velocity. It is known that the three most iron-deficient stars known until now have peculiar enhancement of these intermediate mass elements. Under the assumption that these irondeficient stars reveal the nucleosynthesis result of Pop III stars, we show that the weak supernova model successfully reproduces the observed abundance patterns. Moreover, we show that the initial parameters of the progenitor can be constrained by the model. The initial parameter of Pop III stars is of prime importance for the theoretical study of the early universe. Future observation will increase the number of such carbon enhanced iron-deficient stars, and the same analysis on the stars may give valuable information for Pop III stars that existed in our universe.

\section{References}

[1] Christlieb, N., Bessell, M. S., Beers, T. C., et al. 2002, Natur, 419, 904

[2] Frebel, A., Aoki, W., Christlieb, N., et al. 2005, Natur, 434, 871

[3] Keller, S. C., Bessell, M. S., Frebel, A., et al. 2014, Natur, 506, 463

[4] Hirano, S., Hosokawa, T., Yoshida, N., et al. 2014, ApJ, 781, 60

[5] Stacy, A., Greif, T. H., Klessen, R. S., Bromm, V., \& Loeb, A. 2013, MNRAS, 431, 1470

[6] Takahashi, K., Umeda, H., \& Yoshida, T. 2014, ApJ, 794, 40

[7] Aoki, W., Frebel, A., Christlieb, N., et al. 2006, ApJ, 639, 897

[8] Frebel, A., Christlieb, N., Norris, J. E., Aoki, W., \& Asplund, M. 2006, ApJL, 638, L17

[9] Frebel, A., Collet, R., Eriksson, K., Christlieb, N., \& Aoki, W. 2008, ApJ, 684, 588

[10] Bonifacio, P., Caffau, E., Venn, K. A., \& Lambert, D. L. 2012, A\&A, 544, A102

[11] Christlieb, N., Gustafsson, B., Korn, A. J., et al. 2004, ApJ, 603, 708

[12] Bessell, M. S., Christlieb, N., \& Gustafsson, B. 2004, ApJL, 612, L61

[13] Bessell, M. S., \& Christlieb, N. 2005, in IAU Symp. 228, From Lithium to Uranium: Elemental Tracers of Early Cosmic Evolution, ed. V. Hill, P. Francois, \& F. Primas (Cambridge: Cambridge Univ. Press), 237

[14] Collet, R., Asplund, M., \& Trampedach, R. 2006, ApJL, 644, L121 